\title{
Les gestes dans des réunions de conception architecturale
}

Gestures in architectural design meetings

\section{Willemien Visser}

\section{OpenEdition}

1 Journals

Édition électronique

URL : http://journals.openedition.org/activites/2590

DOI : 10.4000/activites.2590

ISSN : 1765-2723

\section{Éditeur}

ARPACT - Association Recherches et Pratiques sur les ACTivités

\section{Référence électronique}

Willemien Visser, "Les gestes dans des réunions de conception architecturale », Activités [En ligne], 8-2 | octobre 2011, mis en ligne le 15 octobre 2011, consulté le 01 mai 2019. URL : http:// journals.openedition.org/activites/2590; DOI : 10.4000/activites.2590

\section{(9) $\odot \Theta \Theta$}

Activités est mis à disposition selon les termes de la licence Creative Commons Attribution - Pas d'Utilisation Commerciale - Pas de Modification 4.0 International. 


\title{
Les gestes dans des réunions de conception architecturale
}

\author{
Willemien Visser \\ CNRS (UMR 5141, LTCI) Télécom ParisTech - INRIA, France \\ Télécom Paris Tech \\ 46 rue Barrault, B429 - F 75634 Paris Cedex 13 \\ willemien.visser@telecom-paristech.fr
}

\begin{abstract}
Gestures in architectural design meetings. The importance of other modes of expression and representation in the interaction than the "verbal" has been recognised in the field of cognitive design research. With few exceptions, however, the only "nonverbal" modality studied has been graphic, gesture having received little attention.

Our long term goal is to determine the contribution of each of the different semiotic systems that people working on a task adopt in their interaction (especially, speech, graphic, and gestural modalities). Here, we focus on gesture in collaborative design. We analysed an architectural design meeting.

Our research led us to consider design as the construction of representations. In our previous studies of collaborative design, we distinguished representational and organisational activities. These studies were, however, based primarily on the verbal.

In the analysis presented here, we observed that gesture also has these two uses. It contributes to the construction of representations of the artefact and to the organisation of design activities and of interaction among participants.

For a number of authors in the field of gesture research, speech and gesture are an integrated system. Our analysis confirms that gestures are mainly co-verbal.

In conclusion, we discuss the results with respect to possible implications for different collaborative work situations and to their contribution to gesture studies and to cognitive design research.
\end{abstract}

KEYWORDS

Gesture, multimodal interaction, collaborative design, representation, cognitive design research, architecture

\section{1.- Introduction}

L'importance de l'utilisation d'autres modalités d'expression et de représentation que le « verbal» a été reconnue largement dans des études sur la conception dans le domaine du « cognitive design research» (domaine qui englobe, mais dépasse celui de l'ergonomie cognitive de la conception; ne voyant pas de traduction appropriée pour sa dénomination, nous gardons le terme anglais). À 
quelques exceptions près, ce n'est toutefois que le graphique qui a fait l'objet d'études en tant que modalité d'expression et de représentation «non verbale ». L'utilisation du geste a été peu examinée dans ce contexte (des modalités comme le paraverbal, la posture, ou encore les mimiques l'ont été encore moins; dans Visser, 2010a, nous avons commencé l'analyse du regard et de la posture dans une réunion de conception de logiciel). Il est pourtant connu aujourd'hui dans le domaine de la pragmatique interactionnelle que les gestes jouent un rôle essentiel dans la communication, l'interaction et la collaboration (par ex., Goodwin, 2003; Kendon, 2004 ; Mondada, 2004).

L'objectif à long terme de nos recherches dans le domaine de l'interaction est de déterminer la contribution relative de chacun des différents systèmes sémiotiques (oral, écrit, graphique, gestuel et autres modalités d'expression et de représentation) et leur articulation dans l'interaction entre des personnes dans des situations de collaboration. Dans ce texte, nous nous focalisons sur les gestes dans la conception collaborative. Pour l'analyse, nous utilisons des données vidéo recueillies dans une réunion de travail colocalisée entre architectes, travaillant en face à face. À l'aide d'exemples tirés de cette réunion, nous présenterons les utilisations et types de gestes, les différentes formes qu'ils peuvent prendre et les différentes manières dont ils s'articulent avec la parole et le dessin.

\section{1.-Les « gestes » analysés dans notre étude}

Des études sur la communication et l'expression humaines ont montré que l'on n'utilise pas seulement des gestes dans des situations où l'on est face à face avec d'autres personnes ou visible par elles. Par exemple, les gens en utilisent également au téléphone (Bavelas, Gerwing, Sutton, \& Prevost, 2008) et des personnes aveugles de naissance font aussi des gestes, même si elles savent qu'elles s'adressent à une autre personne malvoyante (Iverson, \& Goldin-Meadow, 1997). Cet article ne porte toutefois que sur des gestes utilisés dans l'interaction dans des réunions de travail, car l'étude présentée concerne la conception collaborative (dans une réunion où les concepteurs étaient en face à face).

En outre, les gestes que nous analysons ici sont un sous-ensemble des gestes, souvent qualifiés de « gesticulations » (Kendon, 2004; McNeill, 1992, 2006). Il s'agit des gestes les plus fréquents, en tout cas entre personnes entendantes. Suivant une pratique courante, nous qualifions ces « gesticulations » de « gestes » dans la suite de ce texte. Nous adoptons la définition donnée par McNeill (1992; 2006) : les gesticulations sont des «mouvements qui incarnent du sens en rapport avec la parole qui va avec... Ils sont effectués principalement avec les bras et les mains, mais ne sont pas limités à ces parties du corps » (McNeill, 2006). Elles sont coexpressives avec la parole et sont synchronisées avec celle-ci - même si les deux ne se manifestent pas toujours exactement au même moment (ce point sera discuté plus loin).

On peut rapprocher cette définition de celle donnée par Kendon (2004) dans son livre dont le titre résume la vue de l'auteur : «Geste. De l'action visible comme énoncé » (Gesture. Visible action as utterance). Pour Kendon, le terme «énoncé » « renvoie à tout ensemble d'actions qui comptent pour les autres comme une tentative de l'acteur de 'donner' de l'information de quelque sorte que ce soit » (Kendon, 2004, p. 7).

À côté des gesticulations, il y a des gestes pantomimiques ou, entre personnes sourdes et muettes et certains malentendants, les signes d'une langue des signes (telle que la LSF, la langue des signes française). Les gestes pantomimiques ne sont généralement pas accompagnés de parole; les signes d'une langue des signes sont, par définition, sans accompagnement verbal.

Il y aussi les « emblèmes », des signes de la main conventionnels avec une signification bien établie, comme le geste du pouce levé traduisant l'appréciation positive. S'ils peuvent ou pas être accompagnés de parole, ordinairement ils ne le sont pas et leur signification est indépendante de la parole qui les accompagne éventuellement. 


\section{2- Organisation du texte}

La section suivante présente brièvement quelques orientations de recherche sur les gestes. Après la section 3, sur le rôle des gestes dans la conception collaborative identifié dans des études antérieures, une quatrième section présente les données utilisées dans l'analyse qui sous-tend l'étude présentée ici. Dans les sections 5 et 6 , nous présentons les résultats : d'abord, les différents types de gestes utilisés dans la conception collaborative, ensuite les différentes formes d'articulation de geste et parole. Dans la conclusion, nous formulerons des questions évoquées par notre étude et discuterons les apports de celle-ci aux études sur le geste et à la cognitive design research.

\section{2.- La recherche sur les gestes (angl. gesture studies)}

Il y a de nombreuses perspectives en présence dans ce domaine. Nous nous restreignons ici aux études sur l'utilisation de gestes dans un contexte de travail, pour nous focaliser dans la section suivante au domaine de travail que nous avons étudié, à savoir la conception collaborative.

De plus en plus d'auteurs adoptent un point de vue inspiré par l'approche ethnométhodologique et l'analyse conversationnelle (Sacks, 1995), la micro-ethnographie (Streeck, 2009) ou l'analyse du contexte (Kendon, 2004) - trois perspectives proches auxquelles nous renvoyons, dans ce qui suit, par l'appellation « approche (d'inspiration) ethnométhodologique ».

Ce point de vue peut être illustré à partir d'analyses de «pointage ». Il s'agit du «type » de geste analysé probablement le plus dans les études sur les gestes (Antinoro Pizzuto \& Capobianco, 2008; Bauer, Kortuem, \& Segall, 1999; Enfield, Kita, \& de Ruiter, 2007; Harper, Loehr, \& Bigbee, 2000 ; Kita, 2003a, 2003b; Pizzuto \& Capobianco, 2005; Roussel \& Nouvel, 1999; Zemel, Koschmann, LeBaron, \& Feltovich, 2005). Une analyse de gestes de « pointage » dans une optique ethnométhodologique diffère beaucoup de l'approche « classique » de ces gestes. Adoptant celle-ci - fréquente en IHM - les auteurs analysent les gestes de pointage comme simplement (N.B.) garantissant un partage de la référence à des objets mentionnés dans le discours verbal. Dans les études d'inspiration ethnométhodologique, les auteurs combinent généralement une telle analyse référentielle des déictiques avec une perspective interactionnelle, intersubjective - et ils montrent souvent combien les deux sont dépendantes. Les « déictiques » sont des expressions dont l'interprétation dépend de la situation d'énonciation. Les exemples classiques sont des termes de la langue comme «ici » ou « cela ». Dans le domaine des gestes, l'exemple classique en est le geste de pointage, mais elles peuvent aussi prendre d'autres formes (voir ci-dessous, exemples 2 et 3 ).

Streeck (2009) note ainsi combien l'appellation « pointer » renvoie à des activités complexes. « Des gestes de pointage et d'autres actes physiques d'orientation ne sélectionnent pas simplement des entités physiquement présentes, mais évoquent souvent leurs significations implicites, collectivement connues. Ce sont leurs connaissances partagées du paysage cognitif qui rendent les participants en présence capables de communiquer via un ensemble minimal de gestes d'orientation » (p. 63). Streeck (2009) continue en expliquant qu'il préfère utiliser l'appellation « gestes d'orientation ». Leur but n'est pas tellement de décrire, mais « d'exprimer des relations intentionnelles..., c'est-àdire des configurations dans lesquelles soi-même et autrui établissent un rapport avec l'autre et avec l'objet sur lequel porte l'attention dans l'interaction à venir » (p. 64).

Goodwin (2003), dans son analyse de pointage par des archéologues et par une personne aphasique propose d'analyser « le pointage comme une activité située interactive» (p. 219). Dans son étude d'une réunion de travail entre agronomes et informaticiens, Mondada (2004) adopte une approche similaire. Elle analyse les gestes de pointage des participants, assis autour d'une table remplie de documents, cartes et autres. Elle montre que « les locuteurs pointent vers des détails de la carte non seulement pour référer à eux mais aussi pour organiser et rendre visible leur prise de tour ». Elle constate « une imbrication de pratiques de pointage et d'auto-sélection » dans l'organisation des tours de parole (pp. 274-275). Par l'identification du rôle important des ressources multimodales 
dans ces pratiques, Mondada enrichit et étend ainsi les analyses des pratiques de gestion des tours de parole (angl. turn-taking). En effet, «l'analyse des pratiques de construction du tour a jusqu'ici privilégié surtout les ressources syntaxiques et prosodiques... et a surtout porté sur le face à face conversationnel » (p. 275).

Ces auteurs d'inspiration ethnométhodologique soulignent ainsi combien il est important « de ne pas focaliser son analyse exclusivement sur les propriétés de systèmes de signes individuels, mais d'examiner plutôt l'organisation de l'écologie des systèmes de signes qui ont évolué les uns conjointement avec les autres » (Goodwin, 2003, p. 239).

À côté des analyses d'inspiration ethnométhodologique (telles que celles de Streeck et autres, présentées brièvement) et les études classiques auxquelles elles s'opposent (telles que les analyses de « pointage » d'Antinoro Pizzuto et autres, citées ci-dessus), il y a encore bien d'autres approches. Un exemple en est les travaux de Clot, Fernandez et Scheller (2007). Ces auteurs effectuent leurs études également dans un contexte professionnel et se focalisent sur des gestes avec une visée de travail. Dans l'article cité, les auteurs analysent les gestes de métier dans leur transmission (où le geste transmis est transformé dans une « ré-appropriation » de celui-ci, selon les termes propres des auteurs). Clot et al. (2007) analysent les gestes en tant que composantes d'actions : les actions sont des successions de gestes qui sont orientés par le but des actions. Les auteurs mettent l'accent sur les dimensions sensori-motrices des gestes. L'article porte sur les gestes mis en œuvre dans le tri de lettres sur des casiers de La Poste. Clot et al. (2007) se basent sur des entretiens en auto-confrontation croisée : les auteurs demandent à des professionnels de commenter des images vidéo de l'activité d'un collègue. Ensuite celui-ci commente ces images qui concernent donc sa propre activité. Ces confrontations avec des traces sont suivies de dialogues sur les gestes de métier entre les deux collègues qui, auparavant, ont commenté les images vidéo.

L'attention portée à la réalisation motrice des gestes est un aspect spécifique des études de ces auteurs, qu'ils ne partagent ni avec les auteurs d'inspiration ethnométhodologique, ni avec nous-mêmes. Dans notre intérêt pour la sémantique des gestes, notre optique est beaucoup plus proche des auteurs d'inspiration ethnométhodologique. Dans une étude antérieure, nous avons toutefois analysé la relation entre la fonction et la forme physique des gestes (Visser, 2010b). Notre conclusion était que cette relation n'est pas systématique : des gestes ayant une fonction identique n'ont pas toujours la même forme, et des gestes ayant la même forme n'ont pas invariablement la même fonction.

Pour la majorité des auteurs cités ci-dessus, les enregistrements vidéo constituent les données qui leur permettent leurs analyses. Les auteurs travaillant en IHM ne procèdent pas ainsi habituellement. Clot et al. (2007) utilisent ces enregistrements pour faire effectuer à des professionnels des autoconfrontations. C'est là une dernière spécificité des travaux de ces auteurs que nous avons voulu noter ici. La plupart des auteurs qui s'appuient sur des enregistrements vidéo en font l'analyse euxmêmes, ce qui est aussi notre cas (voir ci-dessous).

Pour une présentation détaillée de la recherche sur les gestes qui couvre le domaine en largeur et commence dans l'antiquité, voir Kendon (2004). Dans les années 60 du xxe siècle, ce chercheur a été lui-même l'un des premiers auteurs modernes à faire des études sur le geste et d'autres formes de communication «non verbale » telles que le regard et la posture (voir Müller, 2007, pour une présentation de l'œuvre de Kendon). En tant que communauté, la recherche sur les gestes n'existe que depuis quelque 20-30 ans (Kendon, 2004). Il s'agit d'un champ interdisciplinaire : les chercheurs viennent notamment de l'anthropologie, la linguistique (en particulier, la pragmatique), la psychologie, la sociologie, la sémiotique, l'informatique (spécialement, l'IHM), les neurosciences, les sciences de la communication, l'histoire (de l'art) et les études sur la musique, le théâtre, la danse et d'autres performances (angl. performance studies). 


\section{3.- L'utilisation du geste dans la conception collaborative : études antérieures}

En comparaison avec l'expression verbale ou graphique, l'utilisation de gestes dans la conception collaborative a été peu examinée. Dans l'une des premières études sur cette activité (conception d'un combiné de commande à distance), Tang (1991) note toutefois que l'expression de nouvelles idées se fait autant de façon gestuelle que graphique ou écrite. Cependant, il note aussi que la gestion de l'interaction est une fonction particulièrement importante des gestes : dans les réunions analysées, plus de la moitié des gestes ont ce rôle.

Quelques années plus tard, Bekker, Olson et Olson (1995) soulignent, dans une analyse de réunions de conception d'un bureau de poste automatisé, l'importance des gestes de caractère spatial, utilisés pour indiquer la taille et l'emplacement d'objets ou la distance entre ceux-ci. Par ailleurs, ils remarquent aussi que, à côté de la conception proprement dite, l'utilisation de gestes peut servir la gestion de la réunion et la régulation du dialogue en général.

Dans une étude monographique d'une équipe d'architectes, Murphy (2005) analyse l'activité de ces concepteurs en termes d'« imagination collaborative». Les architectes observés utilisent des gestes pour imaginer des caractéristiques des entités de conception (leurs mouvement, structure et fonctionnement) et des expériences d'utilisateurs (actions que ces derniers produisent sur les entités de conception).

Par ailleurs, Brassac, Fixmer, Mondada et Vinck (2008), analysant un épisode d'activité collaborative lors d'une réunion sur un projet de mise en œuvre d'un dossier de transfusion informatisé, montrent comment les différentes ressources que sont paroles, gestes, postures et objets sont «entrelacées » (voir le titre de l'article, Interweaving objects, gestures, and talk in context).

Enfin, ces dernières années, de plus en plus d'auteurs en cognitive design research, tout en ne prenant pas spécialement l'utilisation de gestes comme focus de leurs études, l'incluent dans leurs analyses de façon plus ou moins ponctuelle (voir, par exemple, Glock, 2009).

Parmi les fonctions des gestes identifiées dans ces études antérieures, nous soulignerons deux utilisations que nous avons également identifiées comme importantes dans nos analyses. (1) Le geste offre des possibilités spécifiques pour le spatial : d'une part, pour exprimer des qualités spatiales et des qualités ayant un rapport avec le mouvement de l'artefact et, d'autre part, pour représenter des séquences d'action. (2) Le geste a un rôle organisationnel important dans la collaboration.

\section{4.- Données analysées : perspective, provenance, présentation}

Avant de présenter les données sur l'analyse desquelles l'étude décrite ici s'appuie, nous situons brièvement ces données et cette étude dans le contexte de nos travaux sur l'utilisation des gestes dans l'interaction. Ceux-ci sont basés sur des données provenant de deux projets architecturaux. Dans chaque projet, nous avons analysé une réunion conduite dans une étape amont.

Dans la première étude (sur des données recueillies dans le projet MOSAIC, Détienne \& Traverso, 2009), nous nous étions restreintes, en ce qui concerne le geste, au « dessin virtuel » que des architectes effectuent en gesticulant avec une main ou un stylo sur des représentations externes, notamment des plans et des calques (Détienne \& Visser, 2006; Détienne, Visser, \& Tabary, 2006; Traverso \& Visser, 2009; Visser \& Détienne, 2005).

Ce sont les données concernant le second projet que nous utilisons ici. Elles seront présentées cidessous. Dans une analyse antérieure (Visser, 2009a), nous avons examiné ces données en cherchant à identifier la fonction des gestes dans le processus de conception collaborative. En accord avec nos travaux précédents sur la conception, nous avons pu diviser ces gestes en deux grandes familles, gestes représentationnels et gestes organisationnels. 
Par rapport à nos analyses et publications précédentes sur ces données provenant du second projet (Visser, 2009a; 2010b), nous souhaitons introduire ici trois nouveautés : (1) une analyse fonctionnelle plus poussée des gestes représentationnels et organisationnels, prenant en compte d'autres fonctions de ces gestes; (2) une analyse des gestes sur une nouvelle dimension, à savoir, leur articulation avec le verbal (analyse structurelle); (3) une transcription des gestes qui s'ajoute aux descriptions de leur sémantique que nous avions effectuées jusqu'ici.

Analyse des données. C'est en nous appuyant sur notre vue de la conception comme une construction de représentations que nous avons cherché à identifier comment les concepteurs utilisent des gestes (et de la parole) pour construire des représentations de l'artefact (et pour organiser l'interaction et l'activité propre de conception). Il s'agit d'une analyse itérative, procédant par des allers et retours entre des visionnages de la vidéo (les données) et nos transcriptions, descriptions et interprétations.

Provenance des données. Nos données font partie de l'ensemble de données de DTRS7 (le « DTRS7 dataset », Lloyd, McDonnell, Reid et Luck, 2007), mis à la disposition d'une vingtaine de chercheurs invités au $7^{\mathrm{e}}$ Design Thinking Research Symposium (Lloyd, McDonnell, Luck, Reid, \& Cross, 2009). Cet ensemble de données comporte des enregistrements vidéo de quatre réunions de travail entre concepteurs, provenant de deux projets, un projet architectural et un projet d'ingénierie industriel. Nous avons analysé AM1, la 1 1̀re réunion de conception architecturale.

Le dossier de conception (angl. design brief) porte sur la conception d'un crématorium avec une chapelle, placé dans un lieu paysager (comportant déjà un autre crématorium construit dans le passé).

Type et durée de réunion. La réunion était colocalisée; les participants étaient en face à face. Elle a duré $2 \mathrm{~h} 17 \mathrm{~min}$.

Participants. Il y avait trois participants à cette réunion : l'architecte principal chargé du projet, Adam, et deux clients : Anna, l'officier d'état civil des cimetières (angl. registrar) et Charles, un agent du gouvernement local représentant les intérêts de la municipalité (les noms utilisés ici sont des pseudonymes). Une quatrième personne était présente à la réunion : il s'agit de l'un des chercheurs impliqués dans le recueil des données.

Type de données. En ce qui concerne AM1, l'ensemble de données comportait une vidéo de la réunion et la transcription du protocole verbal correspondant; des plans à différentes échelles, élévations, esquisses et projections orthographiques construits et/ou seulement utilisés pendant la réunion; une vidéo avec une interview informelle dans laquelle Adam décrit le contexte du projet (30 minutes).

Transcription verbale. Le protocole verbal transcrit comporte 2342 lignes et 987 tours de parole (c'est-à-dire, des changements de locuteur).

Présentations des données. Nous présenterons des exemples sous forme d'extraits du corpus. Nous faisons précéder ces extraits de leur traduction en français. Ils sont suivis (1) du code de la réunion «AM1», (2) du numéro de ligne dans la transcription et (3) du code identifiant le concepteur («Ad $»=$ Adam; $« \mathrm{An} »=\mathrm{Anna} ; \ll \mathrm{Ch} »=$ Charles $)$.

En dessous de l'extrait présenté ainsi, nous redonnons l'énoncé verbal accompagné, en général, d'une transcription et/ou description des gestes qui l'accompagnent, éventuellement suivie d'une ou plusieurs photos illustrant un ou plusieurs de ces gestes (voir ci-dessous la sous-section Transcription et description des gestes).

La vidéo de la réunion. Elle comporte trois vues : (1) d'une distance lointaine, (2) d'une distance intermédiaire et (3) d'en haut, surplombant la table de travail (Figure 1). 


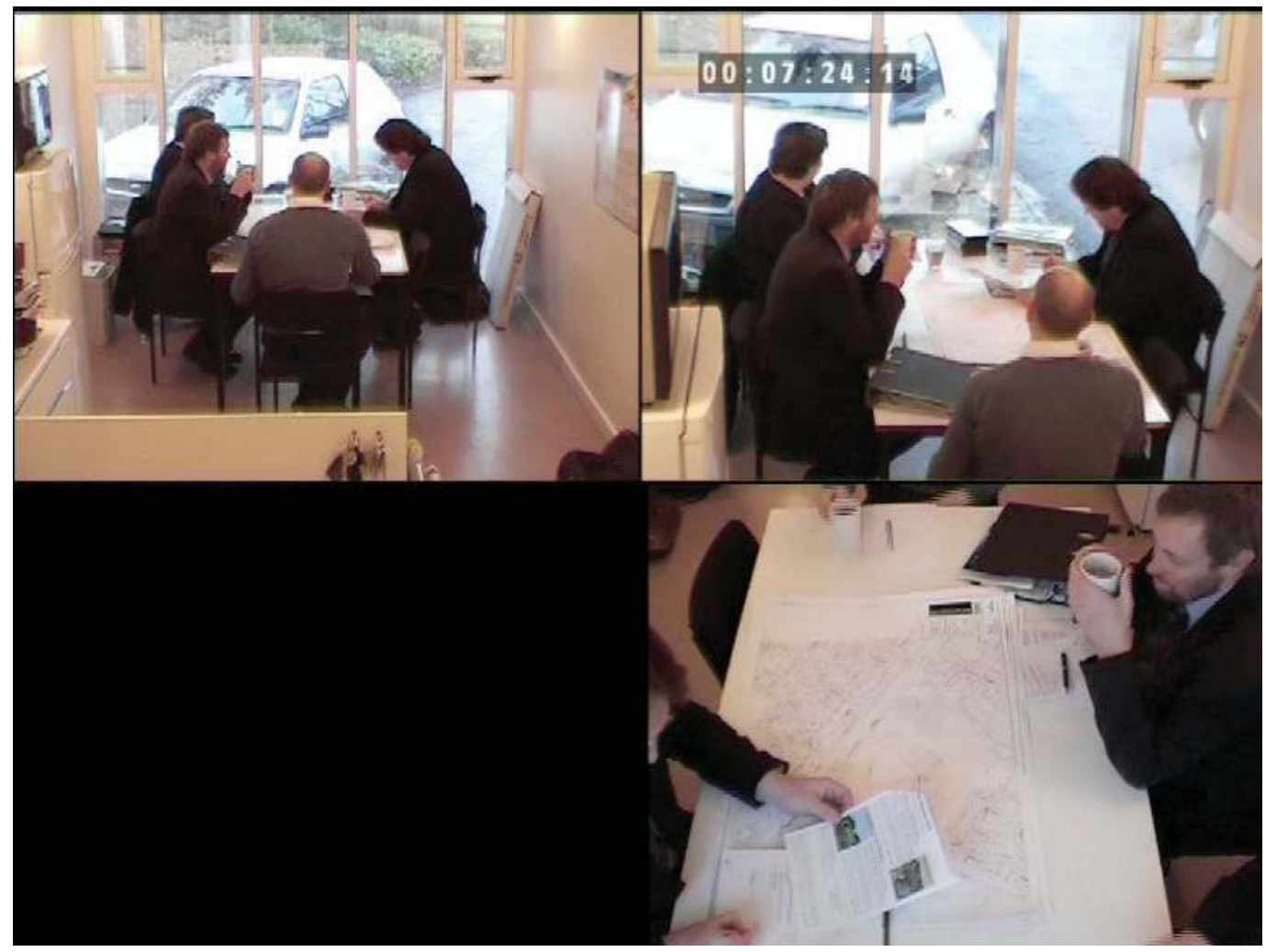

Figure 1 : La vidéo à trois vues de la réunion AM1

Figure 1: The three-view video of the AM1 meeting

Transcription et description des gestes. Comme ce ne sont pas les caractéristiques physiques kinésiques de bas niveau des gestes qui nous intéressent ici, nous transcrivons le geste en décrivant surtout brièvement le mouvement effectué et donnons ensuite, dans le corps du texte, une description de sa sémantique. Nous suivons sur ce point des auteurs comme Kendon (2004), Goodwin (2003) ou Mondada (2004). Nous appliquons les conventions de transcription des gestes développées par Mondada (2008) (notre présentation ci-dessous est basée sur ce document).

En général, nous décrirons en style télégraphique le geste proprement dit «à son apogée, ou extension maximale, où il prend toute sa signification (stroke), qui peut se maintenir plus ou moins longtemps » (Mondada, 2008). Nous utilisons, néanmoins, parfois des symboles pour indiquer certaines caractéristiques des gestes qui nous paraissent importantes à signaler dans leur présentation. Nous suivons, pour ce faire, les conventions proposées par Mondada. L'exemple 1 nous sert à présenter les principaux symboles utilisés dans ces conventions. Pour les quelques autres symboles absents de cet exemple, nous les présenterons et commenterons lors de leur introduction dans des exemples ultérieurs.

Dans cet exemple 1, Adam fait référence, d'une façon assez globale, aux fenêtres de la chapelle (le crématorium proposé par Adam a deux chapelles, une grande « chapelle » et un petit « sanctuaire », dans ce texte « la chapelle » et « le sanctuaire»). Il les caractérise en disant : «bon ce que j'aimerais faire est quelque chose de contemporain peut-être même cubiste ou qui sait une chose qui est contemporaine et architecturale » (well what I'd like to do is something contemporary perhaps even cubist or whatever something that is contemporary and architectural; AM1, 1354-1355, Ad). Dans la minipause qu'Adam laisse après avoir dit "well what I'd like to do is something contemporary", Anna a juste le temps de dire "yeah", avant qu'Adam continue ("perhaps even...") (Figure 2) (Comme le "yeah" d'Anna est absente de la transcription du protocole verbal, nous avons découpé la ligne 1345 du protocole en 1345,1345 ' et $1345 ”)$. 
Exemple 1. Les fenêtres de la chapelle : «j'aimerais faire ... quelque chose de contemporain »
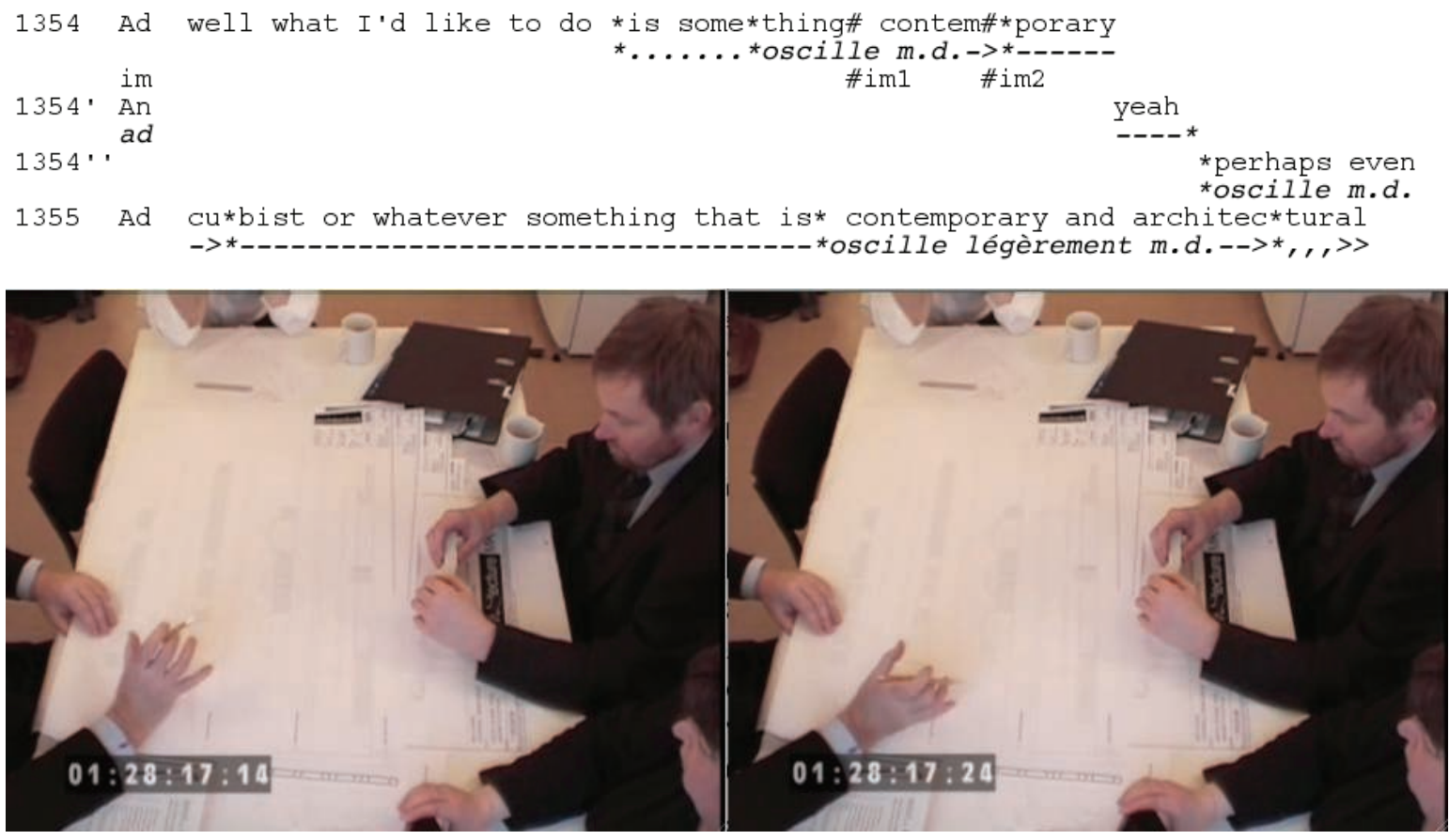

im 1

im2

Figure $2:$ «J'aimerais faire... quelque chose de contemporain ».

Explication de la transcription des gestes

Figure 2: "I'd like to do ... something contemporary"

Explaining the transcription of the gestures

Les gestes sont transcrits entre deux marques de délimitation (* pour Adam), sur une ligne en dessous de la transcription verbale. Leur transcription est alignée spatialement avec la parole correspondante.

Dans l'épisode présenté dans l'exemple 1, Adam commence à gesticuler (au-dessus des « élévations », qui sont les vues que l'on voit dans la Figure 2) en disant « is something contemporary ». Le geste qu'il effectue à trois reprises est une oscillation de sa main droite (m.d.). Avant d'effectuer effectivement ce mouvement, il amorce le geste (........). Suit alors l'apogée du geste, qui est décrit succinctement (ici « oscille m.d.»). Cette description est suivie éventuellement de ----> si le geste continue ou est maintenu pendant une période qui s'étend au-delà de l'espace couvert par sa description écrite (ce qui est le cas ici). Le geste peut ensuite être suspendu dans une position atteinte ou traversée lors de son apogée, noté ---. La personne suspend son geste, dans une position statique : cette immobilité peut être un maintien d'un geste statique quand son apogée est atteint, par exemple, un pointage vers un point bien précis. Une autre possibilité, quand le geste est dynamique, est que le geste est suspendu dans l'une des positions traversées. C'est le cas ici : pendant qu'Adam émet "porary" et pendant qu'Anna dit "yeah", Adam maintient sa main dans la position montrée sur l'image 2 et qui correspond à la « fin » du geste d'oscillation. Le geste peut aussi, après son apogée, décroître de nouveau et la main peut retourner à une position de repos, noté ,,,,,,,. Cette position est souvent (mais pas nécessairement) celle de la main sur la table. C'est le cas ici après le troisième geste d'oscillation (qui est effectué de façon moins prononcée que les deux premiers). Le symbole >> indique que le geste ou autre action transcrit continue jusqu'à la fin de l'extrait et se prolonge au-delà (il est utilisé aussi pour indiquer qu'une action a commencé avant le début de l'extrait, cas dont on verra un exemple plus loin dans le texte). Ici ce symbole transcrit le fait qu'Adam continue à tenir sa main encore sur la table après son émission de "tural". 
Quand ce n'est pas le locuteur qui effectue le geste transcrit, nous indiquons l'identité de la personne qui gesticule dans la marge de la ligne transcrivant le geste. C'est le cas ici d'Adam quand il garde sa main en l'air, à la fin de la trajectoire de son oscillation, pendant qu'Anna dit "yeah". Dans un tel cas, les initiales du participant qui gesticule sont indiquées en minuscules («ad » au lieu d' « Ad»).

Nous essayons en plus de donner une image des gestes par des captures d'écran. Dans ce cas, \#im1 et \#im2 sur une troisième ligne, avec « im » dans la marge, repèrent le moment auquel correspond l'image, de façon synchronisée avec la parole (moment marqué d'un \# dans la transcription du verbal).

Même si nous avons présenté l'exemple 1 pour introduire les conventions de transcription des gestes, nous commentons brièvement les gestes qu'Adam y effectue.

C'est donc en parlant des fenêtres de la chapelle qu'Adam a le discours présenté ici. Nous interprétons ses gestes d'oscillation comme exprimant le caractère approximatif de la caractérisation des fenêtres comme « contemporain[es] peut-être même cubiste[s] » (nous ne comprenons pas leur caractérisation comme « architecturale[s]»).

Nous rapprochons ce geste d'Adam du geste « environ » (angl. thereabouts) décrit par Kendon (2004, pp. 169-170) - à deux différences près : le geste présenté par Kendon est effectué avec les deux mains et les palmes des mains sont parallèles, tandis que la main (droite) d'Adam oscille de façon parallèle à la table, paume ouverte. Kendon décrit ce geste comme exprimant l'idée de "plus ou moins », « approximativement » (Figure 3).

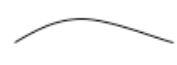

(a)

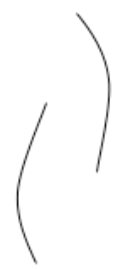

(b)

Figure 3 : Trajectoires (a) de la main d'Adam, oscillante parallèlement à la table et (b) des deux mains décrites par Kendon (2004, pp. 169-170) dans le geste "thereabouts"

Figure 3: Trajectories followed (a) by Adam's hand, oscillating parallel to the table and (b) by the two hands described by Kendon (2004, pp. 169-170) in the "thereabouts" gesture

Kendon qualifie ce geste de «modal » : «il exprime l'attitude de l'énonciateur envers quelque chose, il renvoie à son estimation ou évaluation de quelque chose, plutôt que de décrire quelque chose » (pp. 169-170).

\section{5.- Résultats. Deux types de gestes : représentationnels et organisationnels (analyse fonctionnelle)}

Après une brève introduction concernant notre vision de la conception qui permet de situer en perspective les résultats qui suivent, nous présenterons, dans cette section, les différents types de gestes selon les activités qu'ils accomplissent et/ou dans lesquelles ils interviennent.

\section{1.- La conception comme construction de représentations}

D'un point de vue théorique, nous analysons la conception en termes de construction de représentations (Visser, 2006). 
Le modèle classique en cognitive design research, hérité de Simon (1969/1996), analyse la conception comme une activité de résolution de problèmes - ce que la conception est en effet, d'un point de vue formel. Toutefois, une vision et une analyse de la conception en termes de construction de représentations permettent, à notre avis, de mieux rendre compte de la richesse des activités et structures mises en œuvre dans la conception (Visser, 2006). Cette vue est proche de celles d'auteurs comme, par exemple, Schön (1983) et Glock (2009). Glock voit la conception comme autant de processus sociaux d'interprétation. Pour nous, l'interprétation est une construction de représentations. Dans nos travaux, la (ré)interprétation est l'une des nombreuses activités qui interviennent dans la construction de représentations (à côté d'activités comme l'association, l'analyse, l'exploration, l'inférence, la restructuration, la combinaison, la formulation d'hypothèses, la justification, le raisonnement analogique - et dans d'autres registres, d'une part, l'imagerie mentale et, d'autre part, le dessin (Visser, 2006; voir Visser, 2009b, pour un texte français présentant cette vue de la conception).

C'est sur la base de données verbales et, dans une moindre mesure, graphiques, que nous avions élaboré cette vision de la conception en termes de représentation. Nos analyses de la conception collaborative avaient conduit à y distinguer deux grandes familles d'activités, représentationnelles et organisationnelles. Comme annoncés déjà ci-dessus, nous avons retrouvé ces deux types d'utilisation dans nos travaux sur l'utilisation des gestes dans des réunions de conception architecturale. En effet, nous avons observé que les gestes peuvent, d'une part, contribuer à la construction de la représentation de l'artefact (par la désignation et par la spécification d'entités de conception) et, d'autre part, avoir une fonction organisationnelle (gestion des activités représentationnelles - la tâche de conception « propre » - et organisation de l'interaction).

\section{2.- Construire une représentation de l'artefact à travers des gestes}

L'utilisation de gestes pour la construction d'une représentation de l'artefact - qu'elle soit interne ou externe - peut prendre des formes diverses, en ce qui concerne l'utilisation, forme physique et mode de production des gestes et les types d'attributs de l'artefact représentés gestuellement. Après une brève présentation de la fonction désignationnelle des gestes, nous présenterons différentes formes de l'autre utilisation représentationnelle des gestes, à savoir, celle qui consiste à spécifier des entités de conception en représentant gestuellement l'une ou plusieurs de leurs qualités. Ces qualités peuvent être des attributs spatiaux de l'artefact; elles sont plus ou moins circonscrites, concrètes ou statiques. Nous décrirons ensuite différentes techniques de représentation, d'une part, pour des qualités concrètes, visuospatiales et, d'autre part, pour des qualités abstraites. Avant de passer à ces résultats, nous présenterons brièvement les typologies classiques de gestes; à la fin de cette soussection, nous reviendrons sur ces typologies sur la base de nos résultats.

\subsection{1.- Typologies classiques de gestes}

Les typologies et classifications sont nombreuses dans le domaine de la recherche sur les gestes. Un schéma de classification repris souvent est celui proposé par McNeill et Levy (1982), connu surtout du livre de McNeill (1992). Celui-ci distingue les «déictiques », les « iconiques », les « métaphoriques » et les « gestes de battement» (angl. beats; voir ci-dessous, pour un exemple). En dehors de ce schéma, McNeill (1992) cite aussi les « emblèmes » (voir ci-dessous, l'exemple de «Stop! »). McNeill regroupe les « iconiques » et les «métaphoriques » en « gestes représentationnels ».

\subsection{2.- Représentation de l'objet du discours}

Les gestes sont particulièrement utiles - et utilisés fréquemment - pour désigner l'objet du discours, le « ce dont on parle ». Les participants y font souvent référence en l'indiquant verbalement à l'aide de déictiques, comme « ici » (here) et « cet / cette» (this) (voir exemples 2 et 3 ).

Dans la littérature sur les gestes, les déictiques sont présentés souvent comme étant des «pointages ». Ils peuvent prendre, toutefois, différentes formes. Dans l'exemple 2, Adam utilise un geste de couverture d'une surface avec une main comme déictique; dans l'exemple 3, il effectue en effet 
un pointage prototypique avec un doigt qui pointe un objet (Figures 4 et 5).

Exemple 2 « les gens peuvent traîner ici » (people can mill around here; AM1, 200-201, Ad)

\begin{tabular}{|c|c|c|}
\hline 200 & Ad & \#*people \#can mill \\
\hline & im & $\begin{array}{l}>*^{*} \text { couv. et contourn. } \\
\# \text { im } 1\end{array}$ \\
\hline 01 & im & $\begin{array}{l}\text { \#around here } \\
\text { avec crayon>> } \\
\text { \#im3 }\end{array}$ \\
\hline
\end{tabular}

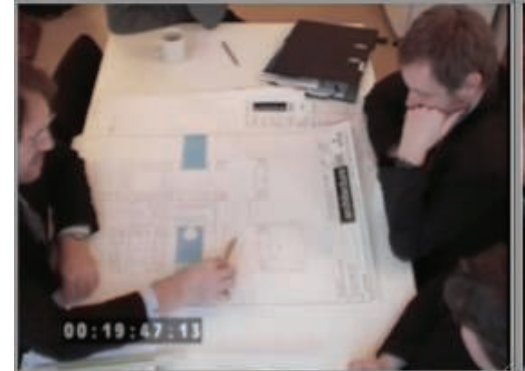

im1

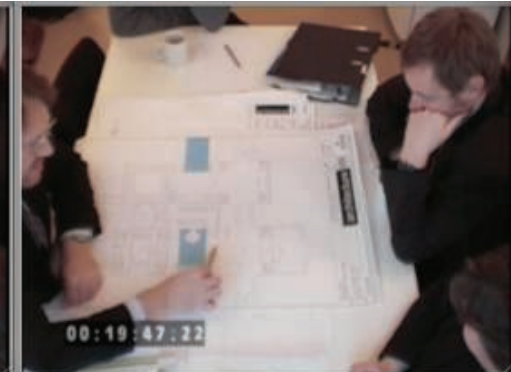

im2

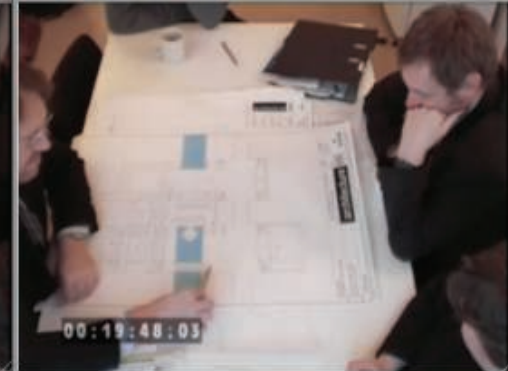

im3

Figure 4 : « les gens peuvent traîner ici »

Figure 4: "people can mill around here"

Pendant qu'il dit "people can mill around here", Adam couvre et contourne avec son crayon la zone où les gens peuvent traîner.

Exemple 3 « [les gens peuvent] regarder cette zone» ([people can] look at this area; AM1, 202, Ad)
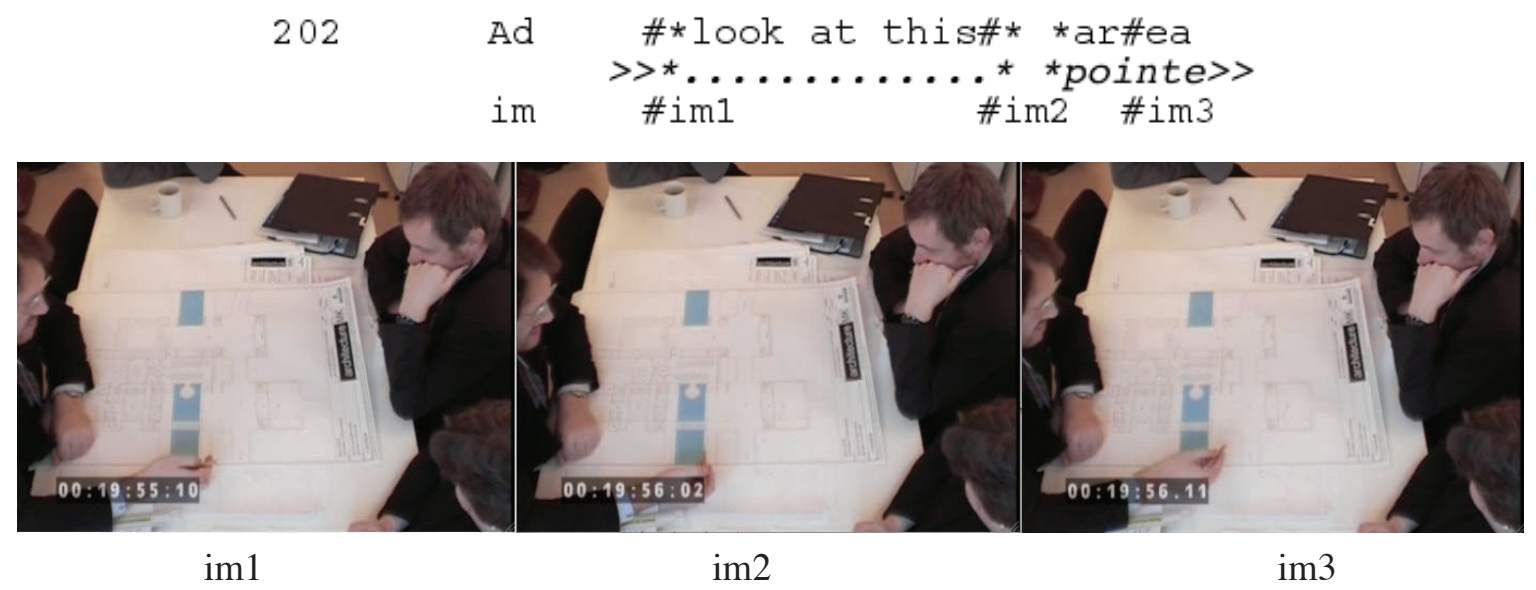

Figure $5:$ « [les gens peuvent] regarder cette zone »

Figure 5: "[people can] look at this area"

Pendant qu'il dit "look at this", Adam prépare son geste de pointage, tournant son crayon dans sa main. C'est en prononçant "area" qu'il indique nettement, en pointant avec son crayon, la zone que les gens peuvent regarder (geste qui continue jusqu'à la fin de l'extrait et se prolonge au-delà).

Ces deux exemples permettent de montrer clairement, d'un côté, qu'un geste déictique peut ne pas être un «pointage » avec un doigt, crayon, stylo ou autre objet pointant (exemple 2) et, d'un autre côté, qu'un tel pointage peut servir à indiquer non seulement un point bien précis, mais aussi une zone (exemple 3).

Des énoncés comportant un déictique verbal sont accompagnés, en général, d'un geste déictique, nécessaire pour que les personnes qui écoutent - et peuvent voir - puissent identifier le référent en question. C'est toutefois aussi parce qu'un énoncé est fait de parole et de gestes qu'un locuteur peut «se permettre » d'utiliser des déictiques verbaux. 
On a vu ci-dessus que les déictiques ne sont, en général, pas utilisés « simplement » pour désambiguïser les référents des objets mentionnés dans le discours verbal. Nous avons noté que les études d'inspiration ethnométhodologique notamment ont souligné que la fonction référentielle des déictiques se combine avec des fonctions interactionnelles. Ici, en indiquant les zones où les gens peuvent traîner, d'une part, et qu'ils peuvent regarder, d'autre part, Adam fait (surtout) une proposition à ses clients. Quelques secondes plus tard, Anna réagit en disant que « les directeurs des entreprises de pompes funèbres se font quelques soucis à ce sujet concernant l'accès pour leurs corbillards ».

Concevoir à travers la désignation. Les gestes de désignation peuvent aussi avoir une fonction de conception " propre » : un concepteur peut concevoir une entité à travers la désignation de celle-ci (angl. design through designation, Visser, 2009a). L'exploitation de cette possibilité est particulièrement flagrante (notamment pour une chercheuse qui analyse les données après coup) dans le cas d'entités qui ne reçoivent pas de représentation explicite sur les documents de conception, tels que le sont en architecture les plans et autres dessins. Il en va ainsi d'entités comme des parcours à travers le bâtiment et autres formes d'utilisation de celui-ci. Par exemple, Adam dit à ses clients qu' « il y a trois parcours possibles [pour aller au « committal room »'] ils passent ou bien par ici un ou ils vont par ici deux ou ils peuvent aller par là trois » (there are three possible routes [to go to the committal room] they either come through here one or they go through here two or they can go through there three; AM1, 1013-1014, Ad; cf. aussi l'utilisation des déictiques verbaux). La description des gestes dynamiques représentant un tel itinéraire se fera dans la section 5.2.6. Caractère plus ou moins statique de l'entité représentée. En formulant cet énoncé, il se peut qu'Adam décrive trois parcours conçus auparavant, comme il se peut qu'il les conçoive sur le champ, au moment que sa cliente Anna se fait des soucis quant à l'accès au "committal room".

\subsection{3.- Représentation d'attributs spatiaux de l'artefact}

Dans la conception architecturale - ainsi que dans la conception mécanique et dans d'autres domaines de conception relatifs à des objets matériels -, la représentation spatiale de l'artefact est centrale. Comme nous l'avons souligné dans la présentation des études antérieures, les gestes se prêtent particulièrement bien à l'expression d'attributs de l'artefact liés à ses caractéristiques spatiales (notamment 3D), son mouvement ou son utilisation dans l'espace. Ainsi, les dimensions de l'artefact sont spécifiées souvent de façon gestuelle. Tant qu'il s'agit de dimensions plus ou moins précises, ce type d'attributs se laisse bien indiquer de façon verbale uniquement - et c'est ce que nous avons observé dans la réunion AM1. Après avoir mesuré, par exemple, avec sa règle, le diamètre du sanctuaire, Adam annonce qu' "il y a une surface au sol de vraiment sept mètres carrés » (there's a floor area of really seven square metres; AM1, 425, Ad). Cet énoncé verbal n'est pas accompagné de geste.

Dès qu'il s'agit, cependant, d'approximations et/ou d'éléments flous ou dépendants d'appréciations subjectives (voir ci-dessous, par exemple, les attributs métaphoriques), le geste permet plus facilement qu'une expression verbale de « donner une idée » de l'entité en question (cf. les possibilités d'expression ambiguë qu'offrent les gestes observés par Streeck, 2009, Note 1, p. 2; voir aussi la littérature sur les difficultés d'utiliser, dans ce type de situations, des systèmes de CAO ou autres qui demandent une expression précise des éléments spécifiés).

Souvent, des gestes qui désignent le référent sont de nature spatiale. C'est que les référents sont fréquemment un objet situé quelque part sur le plan (représentant leur position dans le bâtiment). On en a déjà vu des exemples ci-dessus, dans la section 5.2.2. Représentation de l'objet du discours (exemples 2 et 3 ).

\subsection{4.- Caractère plus ou moins circonscrit de l'entité représentée}

La référence du discours peut être plutôt globale, mais il se peut aussi qu'elle soit assez bien cir-

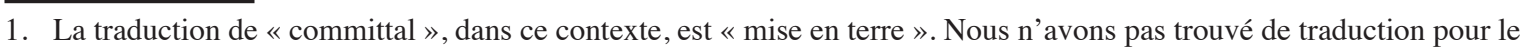
terme « committal room ». Il s'agit d'une salle derrière la chapelle où se trouvent les fours. 
conscrite, comme dans l'exemple donné ci-dessus des « trois parcours possibles » qu'Adam trace du doigt sur le plan. La qualification des fenêtres de la chapelle comme « quelque chose de contemporain peut-être même cubiste ou qui sait... contemporaine et architecturale ", décrite pour présenter la transcription et description des gestes (dans la section du même nom) constitue un exemple de référence plutôt globale.

Un autre exemple en est le suivant. En tant que cliente, Anna dit qu'elle aimerait consulter des utilisateurs (des directeurs d'entreprises de pompes funèbres et, surtout, des personnes qui portent les cercueils). Adam demande alors « est-ce que nous pouvons faire ça rapidement, car pour moi l'idée de cette réunion est d'en partir avec tout ce qui est possible en sachant comment je peux monter en régime le projet pour passer à la planification? » (can we do that soon because the idea of this meeting is for me to go away with what I can knowing how to rev up the design to get it into planning?; AM1, 1204-1206, Ad). En disant cela, il passe avec sa main droite ouverte, doigts étendus, au-dessus du plan, qui représente ainsi le projet global (Figure 6).

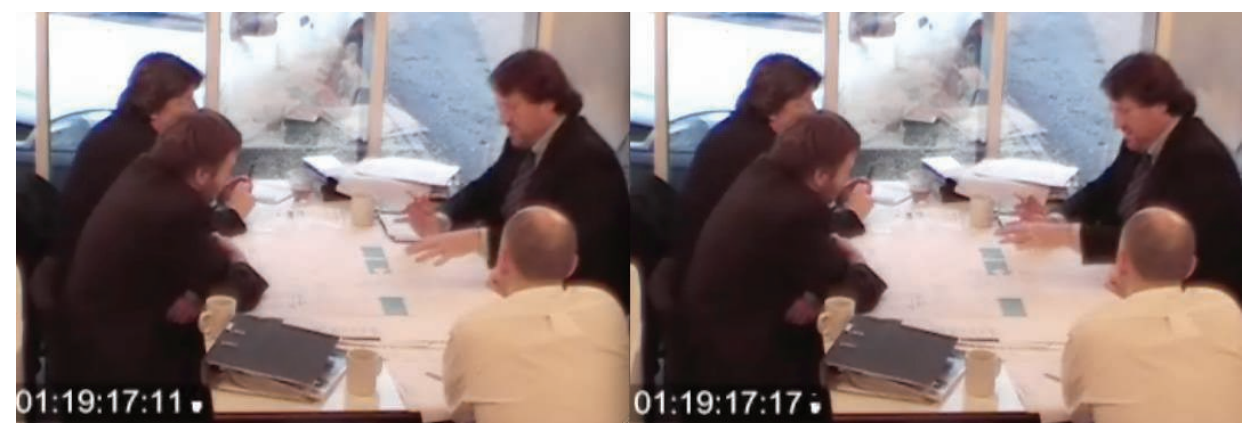

Figure $6:$ «Couverture » du projet global par Adam

Figure 6: "Coverage" of the global project by Adam

\subsection{5.- Caractère plus ou moins concret de l'entité représentée}

L'entité représentée peut être plus ou moins concrète. Dans les exemples des trois parcours et des zones où les gens peuvent traîner ou qu'ils peuvent regarder, elles étaient concrètes; dans celui du projet, elle était quelque peu abstraite - même si le plan utilisé pour y renvoyer était bien concret.

Un autre exemple de référence abstraite est le suivant (exemple 4). Charles affirme que, pour passer l'étang, il faudrait « quelque chose de solide » (something that's solid; AM1, 1178-1179, Ch).

Exemple 4. Pour passer l'étang, il faudrait « quelque chose de solide » (Figure 7).

\begin{tabular}{|c|c|c|}
\hline 1178 & $\mathrm{Ch}$ & $\begin{array}{l}8 \text { something } \\
8 \ldots \ldots \ldots .8\end{array}$ \\
\hline 11 & & $\begin{array}{l}\text { \#8tha\#t's s\#olid } \\
\text { \&A-R au-d. plan>> } \\
\text { \#im1 \#im2 \#im3 }\end{array}$ \\
\hline
\end{tabular}

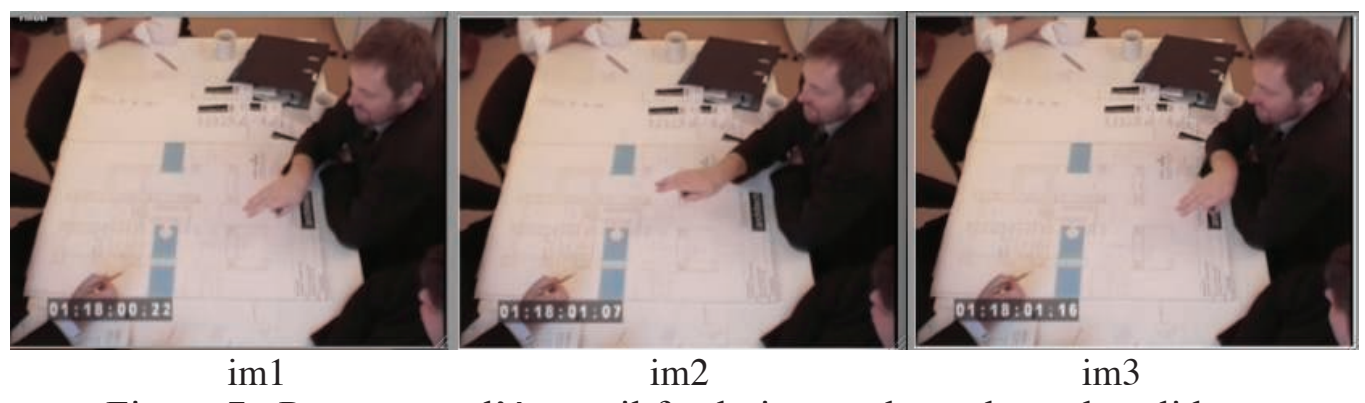

Figure 7 : Pour passer l'étang, il faudrait « quelque chose de solide ».

Figure 7: To cross the pond, one would need "something that's solid"

Pendant qu'il dit "something", Charles amorce son geste, qu'il effectue en disant « that's solid ». Il 
s'agit d'un geste d'une main plate et ouverte, parallèle au plan, qui effectue plusieurs allers-retours au-dessus de et parallèlement à celui-ci, dans un angle d'environ $45^{\circ}$ par rapport au corps de Charles. Passant sa main au-dessus du plan comme s'il traversait l'eau (mais sa main bouge au-dessus du bâti), Charles forme une surface - « quelque chose ». La « solidité » de la surface lui permettant de résister aux efforts (telle qu'un pont) résulte de la tension de la main. Le geste combine des caractéristiques iconiques concrètes (un objet qui traverse l'eau) et abstraites (un objet qui peut résister à des efforts grâce à sa tension).

Un exemple de référence abstraite bien différent est celui d'un simple « ceci » (verbal) renvoyant à une partie du discours énoncée préalablement dans laquelle une entité complexe a été élaborée à l'aide d'un geste. Au moment de son énonciation, le « ceci » est accompagné de ce geste, que seuls les participants ayant été présents à la création de celui-ci peuvent comprendre (observation faite par Becvar, Hollan, \& Hutchins, 2008).

\subsection{6.- Caractère plus ou moins statique de l'entité représentée}

À côté des entités « statiques » présentées dans la plupart des exemples donnés ci-dessus (zone, surface, espace, fenêtres, «quelque chose de solide »), il y a des entités «dynamiques ». Ainsi, les gestes spécifient aussi des mouvements, déplacements et itinéraires - de même que des indications de direction, entités entre statique et dynamique. Ce type d'attributs est d'ailleurs difficile à représenter sans faire appel à des gestes - que ce soit par une description purement verbale de l'artefact ou par un dessin uniquement. Par exemple, décrivant les trois parcours différents qu'il envisage pour que l'on puisse entrer dans la chapelle du crématorium, Adam les trace d'un doigt (geste « déictique » et « iconique »). Figure 8 montre cinq moments dans la description du deuxième parcours (exemple $5)$ : « le deuxième parcours contourne l'étang à son extrémité » (the second route is round the end of the pond; AM1, 1135, Ad).

Exemple 5. « le deuxième parcours contourne l'étang à son extrémité »

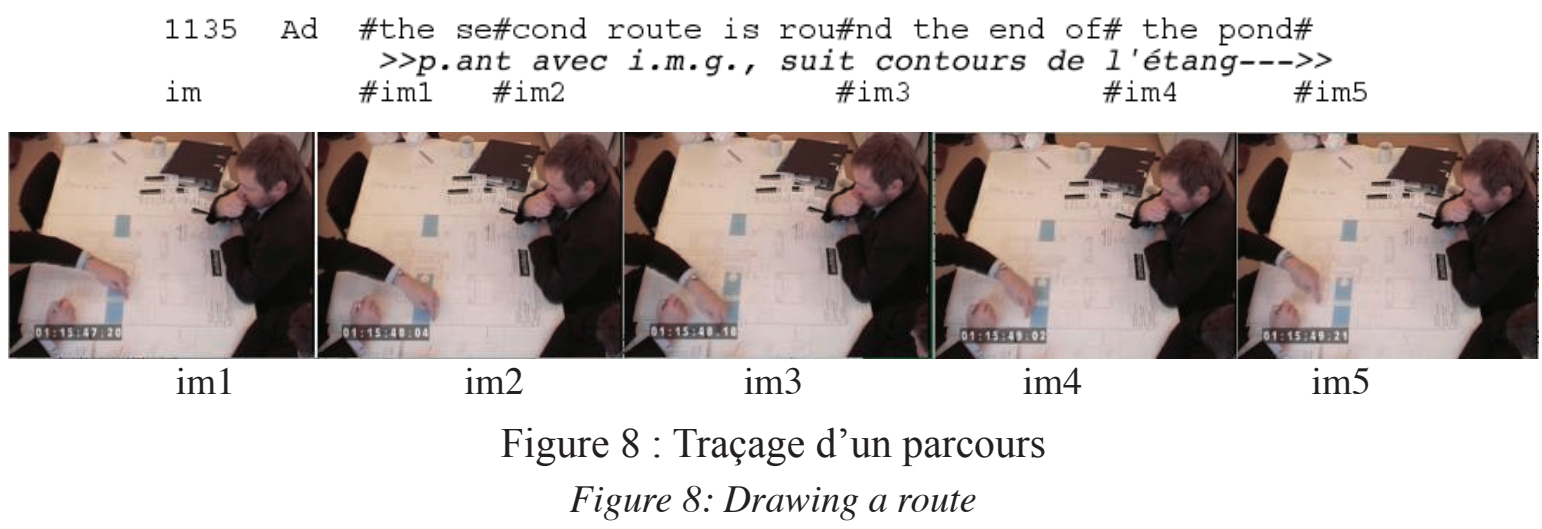

Pointant avec l'index de sa main gauche («p.ant avec i.m.g. »), en disant « le deuxième parcours contourne l'étang à son extrémité », Adam suit les contours de l'étang, indiquant ainsi le deuxième des trois parcours.

\subsection{7.- Différentes techniques de représentation}

Les concepteurs utilisent différentes techniques de représentation dans leur expression gestuelle. Pour des qualités concrètes, visuospatiales (par ex., la forme de l'artefact), nous avons identifié trois techniques :

(1) dépeindre l'artefact en deux dimensions en traçant ses contours avec un doigt ou en le dessinant autrement sur le plan;

(2) modeler l'artefact en le « sculptant » en 3D avec une partie du corps - doigts, main, bras; 
(3) jouer l'utilisation de l'artefact en la mimant.

Pour des qualités abstraites, nous avons observé l'utilisation de gestes métaphoriques. Des propriétés de l'artefact liées à l'atmosphère que celui-ci dégage constituent un exemple de ce type d'attributs. Ainsi, Adam utilise souvent des gestes pour traduire des caractéristiques du bâtiment comme son caractère « audacieux », «pur » ou «calme ». Afin de qualifier, par exemple, le sanctuaire comme « intime», l'architecte enclot avec ses deux mains un espace, comme un adulte qui entoure de ses bras un enfant (exemple 6; im1-im3 de la Figure 9, où le sanctuaire est l'espace rond dans l'étang; un petit passage le relie à la chapelle). La métaphore conceptuelle que le concepteur semble adopter ici est celle de «L'INTIMITÉ EST UN ESPACE CLOS, PROTECTEUR » (cf. ce type de métaphores conceptuelles présentées par Lakoff \& Johnson, 1980).

Exemple 6. « mais si c'est du verre teint-teinté, ce sera un espace beaucoup plus intime » (but if it's stain-stained glass, it'll be a much more intimate space; AM1, 487-488, Ad)

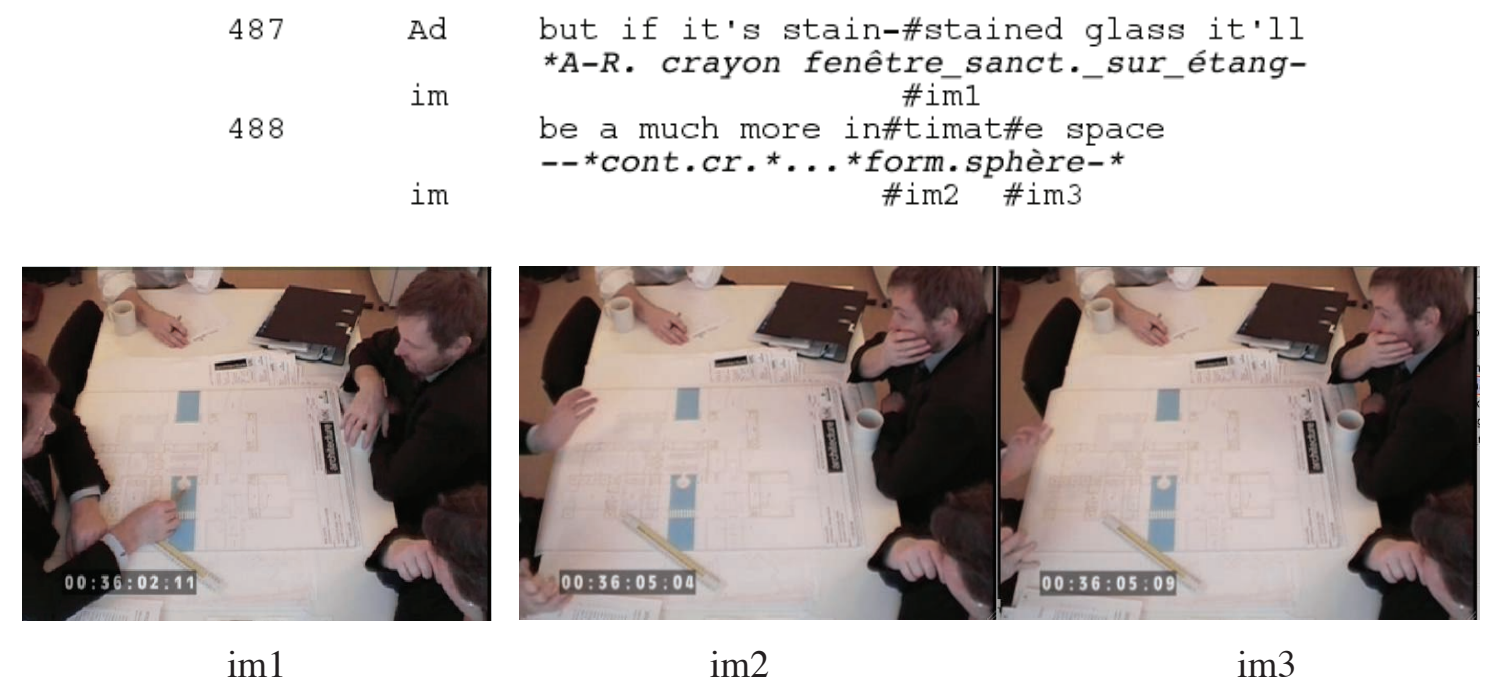

Figure 9 : Geste métaphorique : un espace « intime »

Figure 9: Metaphoric gesture : an "intimate" space

Pendant qu'il dit "but if it's stain-stained glass, it'll be", Adam fait des allers-retours avec son crayon le long de la fenêtre du sanctuaire qui donne sur l'étang (im1). Disant "a much more", il " pointe » le sanctuaire d'un geste qui fait le tour de celui-ci, de manière rapide et approximative (il ne reste pas «dans les lignes »). Terminant "more", il met en place ses deux mains (il les monte de la table en les ouvrant) pour le geste qui va suivre. Disant alors "an intimate space", il fait le geste métaphorique décrit ci-dessus : ouvrant ses deux mains, les paumes arrondies, il forme une sphère. Nous interprétons ce geste comme l'expression de l'idée d' « intimité » par le biais de l'espace clos formé ainsi, comme un adulte qui entoure de ses bras un enfant (im2 et im3). Un élément crucial dans cette interprétation est la dynamique du geste qui ne se montre pas sur des images statiques : il y a, dans le mouvement, une douceur certaine.

\subsection{8.- Gestes représentationnels et typologies classiques de gestes}

Pour McNeill (1992), les « gestes représentationnels » regroupent les « iconiques » et les « métaphoriques ». Contrairement à des définitions basées sur une telle classification des gestes, nous renvoyons par l'expression « gestes représentationnels » à des gestes qui ont une même utilisation ou fonction (désignation ou spécification d'entités de conception). Ainsi, un déictique peut aussi être un geste représentationnel (notamment dans son utilisation de conception par la désignation).

On a vu déjà qu'un geste représentationnel peut être « déictique » et « iconique ». Par ailleurs — 
comme on a pu le voir également dans ce qui précède - de nombreux gestes représentationnels ne sont pas clairement «iconiques » ou « métaphoriques ». Un exemple en est donné par le geste d'Adam quand il dit, d'un espace où les voitures avec les cercueils arrivent, « tout ceci est de nouveau sous un toit » (this is all again under a roof; AM1, 1211-1212, Ad). En disant cela, Adam fait, avec sa main gauche, un geste qui forme une coupelle ouverte vers le bas. Ce geste ne représente pas de façon « fidèle » « un toit », mais représente « quelque chose » qui est « au-dessus » d'un espace et qui « enveloppe » et/ou « couvre » celui-ci (Figure 10).

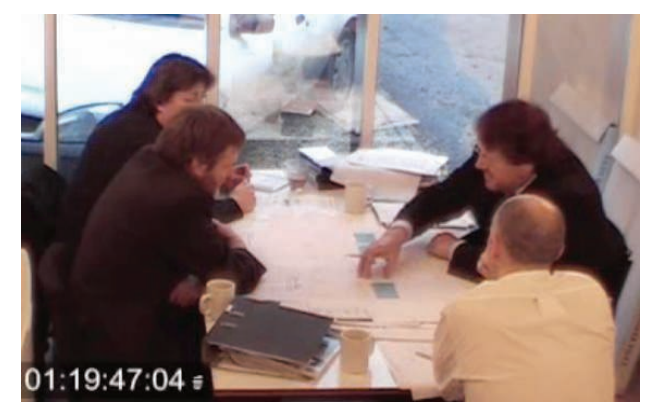

Figure $10:$ «Couverture » d'un espace par « un toit»

Figure 10: "Covering" a space by a "roof"

La classification de McNeill (1992) est encore utilisée beaucoup dans des études en IHM, mais est souvent abandonnée par des chercheurs analysant la richesse des gestes que les personnes font dans des situations de la vie courante, car des distinctions absolues telles qu'il propose sont souvent inappropriées. Dans des publications plus récentes, toutefois, McNeill distingue les gestes plutôt en termes de «dimensions » et parle, par exemple, de l'« iconicité » d'un geste (voir, par exemple, McNeill, 2006).

\section{3.- Organiser la réunion à travers des gestes}

Les concepteurs utilisent aussi des gestes dans un but organisationnel. Nous disposons de moins de résultats sur ce point.

Comme les gestes représentationnels, les gestes organisationnels peuvent prendre de nombreuses formes. Il s'agit de gestes qui contribuent à la gestion de l'interaction (les gestes «interactifs » de Bavelas, Chovil, Lawrie, \& Wade, 1992) et à l'organisation des actions de conception fonctionnelles (la « conception » à proprement parler). Les trois participants à la réunion analysée font usage de gestes organisationnels (mais pas avec la même fréquence; voir ci-dessous notre observation au sujet du caractère idiosyncrasique de l'utilisation de gestes). Pour interrompre les autres et/ou attirer leur attention, ils font, par exemple, le geste «Stop! », en ouvrant la main avec sa palme parallèle au corps, l'ouverture vers $1(\mathrm{es})$ interlocuteur(s) (un geste qualifie souvent d' « emblème »).

Un autre exemple est constitué par des gestes qui modulent le discours. Concernant ces gestes en particulier, le corpus de la réunion montre à quel degré l'utilisation de gestes a des caractéristiques idiosyncrasiques ${ }^{2}$. Anna, en effet, est bien plus expressive que les deux autres participants à la réunion. Souvent elle module son discours de manière particulièrement forte en soulignant ses mots de nombreux gestes bien appuyés. Par exemple, elle ouvre ses deux mains et les étale en face d'elle quand elle dit « j'ai donc fait chercher [ce bouquin] mais tu as beau essayer de les faire passer une commande $\mathrm{SAP}^{3}$, tu reçois en réponse avec quelle fréquence on aura besoin de la maison d'édition Spire » (so I've sent off for [that book] but you try and get them to raise a SAP order for it and you

2. On trouve un autre exemple du caractère idiosyncrasique de l'expression gestuelle dans la réunion analysée dans la première étude, où l'un des trois concepteurs ne fait ses dessins que de facon gestuelle: l'architecte en question fait seulement du « dessin virtuel ».

3. Une « commande SAP » est basée sur SAP, une méthode de gestion d'entreprise. 
get how often will we need the Spire books company; AM1, 39-41, An) (Figure 11).

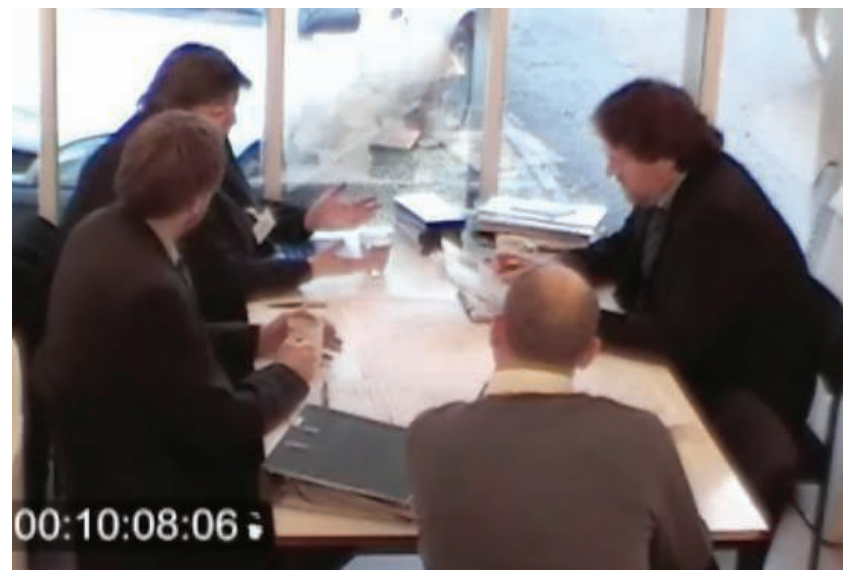

Figure 11 : Modulation gestuelle de son discours par Anna

Figure 11: Gestural modulation of her discourse by Anna

Ce « geste de battement» dans la classification de McNeill (1992) constitue pour Bavelas et al. (1992) un geste interactif de "partage d'information ». Ici, Anna ne partage pas tant une information factuelle que son émotion (irritation) au sujet de celle-ci. Bavelas paraphrase ce geste de « comme vous savez! »-mais les deux autres participants à la réunion ne savent probablement pas autant qu'Anna combien « l'administration » peut vous freiner dans de « simples » commandes.

\section{6.- Résultats. Deux formes d'articulation des gestes et de la parole : intégration ou autonomie (analyse structurelle)}

Nous avons montré ci-dessus comment les gestes peuvent contribuer à la construction de la représentation de l'artefact et à l'organisation de l'interaction et des activités de conception propres. Par rapport au verbal, ils font cela, globalement, de deux manières : dans une combinaison intégrée avec la parole ou de façon autonome, c'est-à-dire sans qu'il y ait une contribution verbale.

Des chercheurs travaillant dans le domaine des études sur le geste ont défendu que geste et parole constituent un système intégré unique (McNeill, 2000). À l'exception de la communication en langue des signes, les gestes sont presque toujours produits dans une combinaison intégrée avec la parole - même si, dans un flux continu de parole et de gestes co-verbaux, il y a des gestes qui ne sont pas accompagnés d'une expression verbale, par exemple, des gestes pantomimiques ou des emblèmes.

Les gestes présentés ci-dessus étaient presque tous co-verbaux. Nous avons identifié peu de véritables gestes « autonomes ». Un geste interactif comme l'emblème « Stop! » en est toutefois un exemple. Production de geste et de parole intégrés peut, toutefois, se faire de façon plus ou moins simultanée.

\section{1.- Caractère plus ou moins concomitant de gestes et parole intégrés}

Même si gestes et parole sont intégrés, les deux ne sont pas systématiquement réalisés en même temps. Un exemple d'un geste qui précède nettement son expression verbale est l'exemple de la métaphore gestuelle représentant « intime», décrite ci-dessus. La représentation de ce type d'attribut abstrait semble difficile à effectuer verbalement. Cette difficulté permet d'expliquer que le concepteur y a recours en premier et/ou plus aisément par rapport au verbal.

McNeill (2006) attribue à des hésitations ce type de gestes avant-coureurs de l'énoncé. Il écrit, en renvoyant à un large échantillon de gestes analysé par Shuichi Nobe (dont il ne donne pas la référence), que « la phase de l'apogée de la gesticulation est synchrone avec le discours co-expressif 
environ $90 \%$ du temps.... Lorsque des gestes à l'apogée sont asynchrones, ils précèdent légèrement le discours auquel ils sont liés sémantiquement, généralement en raison d'hésitations brèves, et l'intervalle en temps est petit. »

\section{2.- Différents types de relations entre gestes et parole intégrés}

Même si un geste est produit de façon co-verbale, son apport peut avoir différents types de liens avec le verbal. Parfois, le geste semble fournir une expression parallèle à ce qui est exprimé en mots : la « même chose » est exprimée par deux modalités différentes. D’autres fois, le gestuel raffine, nuance, précise ou restreint ce qui est représenté verbalement. D’autres fois encore, il fait le contraire : il est global tandis que la parole circonscrit la chose. Il peut même présenter des aspects différents de ce qui est exprimé verbalement : nous n'en avons pas identifié d'exemples, mais d'autres auteurs en ont observé (Cienki \& Müller, 2008; Kendon, 2004, p. 161).

\section{7.- Conclusion}

Le Tableau 1 présente un résumé de nos principaux résultats.

\begin{tabular}{|c|c|c|c|c|c|}
\hline \multicolumn{4}{|c|}{$\begin{array}{l}\text { caractéristiques des gestes au plan fonctionnel : } \\
\text { fonction des gestes }\end{array}$} & \multirow{2}{*}{$\begin{array}{c}\text { réalisation } \\
\text { des gestes } \\
\text { représentationnels }\end{array}$} & \multirow{2}{*}{$\begin{array}{l}\text { caractéristiques } \\
\text { des gestes au plan } \\
\text { structurel : } \\
\text { articulation entre } \\
\text { geste et verbal }\end{array}$} \\
\hline \multicolumn{2}{|c|}{ représentationnelle } & \multicolumn{2}{|c|}{ organisationnelle } & & \\
\hline $\begin{array}{l}\text { désigner } \\
\text { l'artefact }\end{array}$ & $\begin{array}{l}\text { spécifier des } \\
\text { attributs de } \\
\text { l'artefact associés } \\
\text { à } \\
\text { - des qualités } \\
\text { spatiales } \\
\text { - l'utilisation } \\
\text { - l'atmosphère } \\
\text { - d'autres } \\
\text { caractéristiques } \\
\text { de l'artefact }\end{array}$ & \multirow[t]{2}{*}{$\begin{array}{l}\text { organiser les } \\
\text { activités de } \\
\text { conception }\end{array}$} & \multirow[t]{2}{*}{$\begin{array}{l}\text { organiser } \\
\text { l'interaction } \\
\text { entre les } \\
\text { participants }\end{array}$} & \multirow{2}{*}{$\begin{array}{l}\text { techniques de } \\
\text { représentation } \\
\text { - représentation en } \\
\text { deux dimensions } \\
\text { - représentation en } \\
\text { trois dimensions } \\
\text { - représentation à } \\
\text { travers l'utilisation } \\
\text { de l'artefact } \\
\text { - représentation } \\
\text { métaphorique }\end{array}$} & \multirow{2}{*}{$\begin{array}{l}\text { - intégration des } \\
\text { deux } \\
\text { - autonomie } \\
\text {-- geste sans } \\
\text { accompagnement } \\
\text { verbal } \\
\text {-- parole sans } \\
\text { accompagnement } \\
\text { gestuel }\end{array}$} \\
\hline \multicolumn{2}{|c|}{$\begin{array}{lr}\text { conduisant à des entités plus ou } \\
\text { moins } \\
\text { - } & \text { circonscrites } \\
\text { - } & \text { concrètes }\end{array}$} & & & & \\
\hline
\end{tabular}

Tableau 1 : Résultats de l'étude :

Caractéristiques des gestes au plan fonctionnel et au plan structurel

Table 1: Summarised results of the study:

Caracteristics of the gestures from a functional and from a structural vieuwpoint

Notre étude, exploratoire et descriptive, apporte beaucoup de données. Elle évoque aussi de nombreuses questions. Après une discussion d'un certain nombre de ces questions, nous clorons cet article par quelques remarques concernant le double apport de cette étude, d'une part, aux études sur le geste, d'autre part, à la cognitive design research.

\section{1.- Questions évoquées par cette étude}

Une première question concerne l'influence qu'a l'utilisation de gestes sur le processus de collaboration. Cette question nécessiterait des études dans lesquelles on aurait, au moins, deux conditions : collaboration avec et collaboration sans accès au geste - et éventuellement au regard et à d'autres modalités d'expression. Il s'agirait donc d'études expérimentales (cf. les différentes études effec- 
tuées par Perron et par Lefebvre, présentées dans Lefebvre, Perron, \& Pauchet, 2007; Perron, 2005).

Nos données proviennent d'études de terrain, conduites sur des réunions professionnelles entre architectes, colocalisés et travaillant en face à face (nous incluons ici l'étude MOSAIC). Pendant ces réunions, les concepteurs utilisent des plans en papier qu'ils modifient et ils font des croquis sur du papier-calque (les plans « définitifs » sont faits avec des systèmes de dessin assisté par ordinateur, hors réunion). De telles données peuvent inspirer des idées quant à l'utilisation du geste dans des situations de travail collectif à distance où la communication est médiée par l'intermédiaire de systèmes informatiques. Elles montrent que les gestes sont fréquents et ont de nombreuses utilisations différentes. Elles montrent aussi que les gestes ne constituent pas simplement des «illustrations » de ce qui est dit et que l'« on pourrait s'en passer ». Il est possible de formuler des hypothèses prédisant des gestes particulièrement utiles ou même indispensables. Toutefois, la spécification de systèmes informatiques pouvant « médier » dans des situations de collaboration à distance (des systèmes de Travail Coopératif Assisté par Ordinateur, ou, plus généralement, des systèmes de Communication Médiée par Ordinateur) demande, à notre avis, des études spécifiquement dédiées à cette question.

Notre étude ne permet pas d'affirmer que certains types d'attributs s'expriment mieux ou de façon privilégiée de façon gestuelle plutôt qu'en utilisant un autre système sémiotique (verbal, graphique). Nous faisons toutefois l'hypothèse que l'expression de tout ce qui est dynamique ou mobile (par exemple, des itinéraires, ou plus généralement, l'utilisation de l'artefact) se fait de façon plus aisée par des gestes que verbalement - et que cet « avantage » vaut aussi bien pour l'expression que pour la compréhension. Nous conjecturons aussi que les gestes constituent un système privilégié pour l'expression de certains attributs abstraits - tels que l'atmosphère que l'artefact dégage. Ces hypothèses restent à mettre à l'épreuve.

Une autre question concerne l'influence de la langue, et plus largement de la culture des concepteurs. Toujours plus d'entreprises sont le résultat d'une collaboration internationale et leurs projets de conception font appel à des équipes interculturelles (dans l'automobile, par exemple, France et Japon; dans l'industrie aéronautique et spatiale, par exemple, France, Allemagne et Espagne). Dans la réunion analysée, les trois participants sont anglais, mais, «naïvement », on peut noter des différences importantes dans la gestualité entre les trois qui ne semblent pas uniquement idiosyncrasiques (cf. la Note 2). On peut faire des hypothèses «préscientifiques » sur l'origine de ces différences; les conceptualiser et opérationnaliser pour pouvoir les examiner reste toutefois une autre affaire!

\section{2.- Contribution aux études sur le geste}

Jusqu'ici, le geste a été peu étudié dans des situations orientées par un but, telles que celle que nous avons examinée, à savoir une réunion de travail. Notre étude a montré comment les gestes classiques (comme les déictiques, iconiques et autres) sont utilisés avec des fonctions particulières grâce à la tâche spécifique dans laquelle ils sont utilisés - ici la conception.

Nous avons identifié des gestes représentationnels et des gestes organisationnels, ce qui correspond en effet aux principales familles d'activités de conception (Visser, 2006). Des gestes organisationnels se produisent dans chaque activité, mais les gestes représentationnels sont, à notre avis, typiques pour la conception. Parmi ces derniers, nous avons distingué désignation et spécification. Les gestes de spécification peuvent sembler les gestes de conception caractéristiques. Toutefois, si la désignation se fait dans toute interaction, ce n'est pas avec la fonction particulière identifiée ici dans une situation de conception collaborative, à savoir, la conception à travers la désignation. En ce qui concerne la spécification, dans toute interaction les participants spécifient du sens pour les autres afin d'établir une « base commune » (angl. common ground), mais, dans la conception, la spécification ne vise pas seulement l'inter-compréhension, mais aussi l'accord (une distinction que Visser, 2007, introduit dans son analyse de « common ground »). 


\section{3.- Contribution à la cognitive design research}

Cette étude contribue à notre connaissance sur la conception en ce qui concerne les activités qui y sont mises en œuvre et la réalisation physique de ces activités. Pour des aspects des artefacts à concevoir liés à l'espace (en particulier 3D), le mouvement et l'action, elle relève, d'une part, la nature particulière de la façon dont ces aspects peuvent être conçus - à savoir, par différents types d'énoncés multimodaux - et, d'autre part, le caractère particulièrement approprié du système sémiotique du geste pour la mise en œuvre des activités de conception en question. Nos résultats indiquent en effet le rôle essentiel du geste à la fois dans la conception et dans la communication liées au 3D et aux aspects de la conception dynamiques (comme le mouvement et l'action), qui sont difficiles, voire impossibles, à élaborer en n'utilisant que l'expression verbale ou graphique.

Jusqu'à présent, les activités de désignation n'ont pas été abordées spécialement en cognitive design research. La présente analyse pointe l'ampleur de ces activités dans la conception collaborative. Le rôle essentiel du geste pour la deixis était déjà bien connu; ce que nos résultats y ajoutent sont (1) l'importance des activités de désignation avec une fonction de conception (dans la conception collaborative) et (2) la mise en œuvre de cette fonction par le geste.

Même si elle n'est pas spécifique à la conception, nous tenons également à souligner l'importance du geste pour les questions d'organisation. Ce résultat vient confirmer les travaux antérieurs sur le geste dans des réunions de conception. La présente étude, cependant, a montré que cette fonction du geste couvre encore plus que ce qui avait été montré déjà, à savoir également la gestion des actions de conception «propres ».

\section{REMERCIEMENTS}

Nous remercions les participants à la réunion de nous avoir permis de les filmer et d'analyser leur activité. Les données présentées et analysées ici (provenant du «DTRS7 dataset») ne sont pas publiquement disponibles.

Nous remercions également les deux relecteurs de la première version de cet article. Leurs remarques ont permis d'introduire dans cette version définitive des modifications qui nous semblent constituer des améliorations.

Enfin, nous remercions notre correspondante à Activités, Valérie Pueyo, qui nous a fait des suggestions bien utiles de changement.

\section{RÉFÉRENCES}

Antinoro Pizzuto, E., \& Capobianco, M. (2008). Is pointing "just" pointing? Unraveling the complexity of indexes in spoken and signed discourse. Gesture, Special Issue "Dimensions of gesture", 8(1), 82-103.

Bauer, M., Kortuem, G., \& Segall, Z. (1999, October 18-19). "Where are you pointing at?” A study of remote collaboration in a wearable videoconference system. Paper presented at the Third International Symposium on Wearable Computers, San Francisco, CA.

Bavelas, J. B., Chovil, N., Lawrie, D. A., \& Wade, A. (1992). Interactive gestures. Discourse Processes, 15, 469-489.

Bavelas, J. B., Gerwing, J., Sutton, C., \& Prevost, D. (2008). Gesturing on the telephone: Independent effects of dialogue and visibility. Journal of Memory and Language, 58, 495-520.

Becvar, A., Hollan, J., \& Hutchins, E. (2008). Representational gestures as cognitive artifacts for developing theories in a scientific laboratory. In M. S. Ackerman, C. A. Halverson, T. Erickson, \& W. A. Kellogg (Eds.), Resources, co-evolution, and artifacts: Theory in CSCW (pp. 117-143). London: Springer.

Bekker, M. M., Olson, J. S., \& Olson, G. M. (1995). Analysis of gestures in face-to-face design teams provides guidance for how to use groupware in design. Paper presented at DIS95, Conference on Designing interactive systems: Processes, practices, methods, \& techniques, Ann Arbor, MI.

Brassac, C., Fixmer, P., Mondada, L., \& Vinck, D. (2008). Interweaving objects, gestures, and talk in context. Mind, Culture and Activity: An International Journal, 15(2), 208-233. 
Cienki, A. J., \& Müller, C. (2008). Metaphor, gesture, and thought. In J. Raymond \& W. Gibbs (Ed.), The Cambridge Handbook of Metaphor and Thought (pp. 483-501). Cambridge: Cambridge University Press.

Clot, Y., Fernandez, G., \& Scheller, L. (2007). Le geste de métier : problèmes de la transmission. Psychologie de l'Interaction, 23, 109-139.

Détienne, F., \& Traverso, V. (Eds.). (2009). Méthodologies d'analyse de situations coopératives de conception. Nancy: Presses Universitaires de Nancy.

Détienne, F., \& Visser, W. (2006). Multimodality and parallelism in design interaction: Co-designers' alignment and coalitions. In P. Hassanaly, T. Herrmann, G. Kunau, \& M. Zacklad (Eds.), Cooperative systems design. Seamless integration of artifacts and conversations-Enhanced concepts of infrastructure for communication (pp. 118-131). Amsterdam: IOS. Aussi disponible à http://hal.inria.fr/inria-00118255/ en/.

Détienne, F., Visser, W., \& Tabary, R. (2006). Articulation des dimensions graphico-gestuelle et verbale dans l'analyse de la conception collaborative. Psychologie de l'Interaction. Numéro spécial "Langage et cognition: Contraintes pragmatiques”, 21-22, 283-307.

Enfield, N. J., Kita, S., \& de Ruiter, J. P. (2007). Primary and secondary pragmatic functions of pointing gestures. Journal of Pragmatics, 39(10), 1722-1741

Glock, F. (2009, 16-19 July). Designing as interpretation. Paper presented at the Undisciplined! Design Research Society Conference, Sheffield Hallam University, Sheffield, UK.

Goodwin, C. (2003). Pointing as situated practice. In S. Kita (Ed.), Pointing: Where language, culture and cognition meet (pp. 217-241). Mahwah, NJ: Lawrence Erlbaum.

Harper, L., Loehr, D., \& Bigbee, A. (2000, June 12-16). Gesture is not just pointing. Paper presented at the First International Natural Language Generation Conference (INLG'2000), Mitzpe Ramon, Israel.

Iverson, J., \& Goldin-Meadow, S. (1997). What's communication got to do with it? Gesture in children blind from birth. Developmental Psychology, 33, 453-467.

Kendon, A. (2004). Gesture: Visible action as utterance. Cambridge, England: Cambridge University Press.

Kita, S. (2003a). Pointing: A foundational building block of human communication. In S. Kita (Ed.), Pointing. Where language, culture, and cognition meet (pp. 1-8). Hillsdale, NJ: Lawrence Erlbaum Associates.

Kita, S. (Ed.). (2003b). Pointing. Where language, culture, and cognition meet. Hillsdale, NJ: Lawrence Erlbaum Associates.

Lakoff, G., \& Johnson, M. (1980). The metaphorical structure of the human conceptual system. Cognitive Science, 4, 195-208.

Lefebvre, L., Perron, L., \& Pauchet, A. (2007). Animations d'avatars dans les EVC : Choix des indices non verbaux pour l'interaction. Communication présentée aux 2èmes journées de l'AFRV (Association Française de Réalité Virtuelle), Marseille, France.

Lloyd, P., McDonnell, J., Luck, R., Reid, F., \& Cross, N. (2009). About: Designing: Analysing design meetings. London: Taylor \& Francis.

Lloyd, P., McDonnell, J., Luck, Reid, F., \& Luck, R. (2007). DTRS7 dataset. Non disponible au public.

McNeill, D. (1992). Hand and mind. What gestures reveal about thought. Chicago: University of Chicago Press.

McNeill, D. (2006). Gesture: A psycholinguistic approach. In K. Brown (Ed.), Encyclopedia of Language and Linguistics (2 ed., Vol. Psycholinguistics Section). Amsterdam: Elsevier. Accédé le 6 september 2011, du World Wide Web http://mcneilllab.uchicago.edu/pdfs/gesture.a_psycholinguistic_approach.cambridge. encyclop.pdf

McNeill, D. (Ed.). (2000). Language and gesture. Cambridge: Cambridge University Press. 
McNeill, D., \& Levy, E. T. (1982). Conceptual representations in language activity and gesture. In R. Jarvella \& W. Klein (Eds.), Speech, place, and action: Studies in deixis and related topics (pp. 271-295). Chichester, England: Wiley.

Mondada, L. (2004). Temporalité, séquentialité et multimodalité au fondement de l'organisation de l'interaction : Le pointage comme pratique de prise du tour. Cahiers de Linguistique Française, 26, 169192.

Mondada, L. (2008, avril). Conventions de transcription des gestes (version 2.0.7, avr08). Accédé le 27 avril 2011, du World Wide Web : http ://icar.univ-lyon2.fr/projets/corinte/documents/convention_transcription_ multimodale.pdf

Müller, C. (May 2007). A semiotic profile : Adam Kendon. Semiotix. A Global Information Bulletin, Issue 9. Accédé le 21 février 2011, du World Wide Web : http://www.semioticon.com/semiotix/semiotix9/sem-903.html

Murphy, K. M. (2005). Collaborative imagining: The interactive use of gestures, talk, and graphic representation in architectural practice. Semiotica, 156(1/4), 113-145.

Perron, L. (2005, 12-16 septembre). An avatar with your own gestures. Communication présentée au workshop d'Interact'05 “Appropriate methodology for empirical studies of privacy”, Roma.

Pizzuto, E., \& Capobianco, M. (2005, October 21-23). Is pointing just pointing? Paper presented at the International Conference "Il gesto nel Mediterraneo: Studi recenti sulla gestualità nel sud d'Europa (Gesture in the Mediterranean: Recent research in Southern Europe)", Procida, Italy.

Roussel, N., \& Nouvel, G. (1999, novembre). La main comme télépointeur. Communication présentée à IHM99, 11ièmes Journées francophones sur l'Interaction Homme Machine, Montpellier.

Sacks, H. (1995). Lectures on conversation (Vol. I and II. Edited by G. Jefferson with an Introduction by E. A. Schegloff). Oxford: Wiley-Blackwell.

Schön, D. A. (1983). The reflective practitioner: How professionals think in action. New York: Basic Books. (Reprinted in 1995).

Simon, H. A. (1969/1996). The sciences of the artificial (3rd, rev.ed. 1996; Orig.ed. 1969; 2nd, rev.ed. 1981) (3rd ed.). Cambridge, MA: MIT Press.

Streeck, J. (2009). Gesturecraft. The manu-facture of meaning. Amsterdam: John Benjamins.

Tang, J. C. (1991). Findings from observational studies of collaborative work. International Journal of ManMachine Studies, 34, 143-160.

Traverso, V., \& Visser, W. (2009). Co-élaboration de solutions et rôle du graphico-gestuel : confrontation de méthodologies. In F. Détienne \& V. Traverso (Eds.), Méthodologies d'analyse de situations coopératives de conception : Corpus MOSAIC (pp. 87-182). Nancy, France: Presses Universitaires de Nancy.

Visser, W. (2006). The cognitive artifacts of designing. Mahwah, NJ: Lawrence Erlbaum Associates.

Visser, W. (2007, August 28-31). Collaborative designers' different representations. Paper presented at the 16th International Conference on Engineering Design, ICED’07, Design for society. Knowledge, innovation and sustainability, Paris (Proceedings CD : ISBN 1-904670-02-4; Abstracts of papers : ISBN 1-904670-01-6, pp. 449-450).

Visser, W. (2009a). The function of gesture in an architectural design meeting (ch. 15). In J. McDonnell \& P. Lloyd \& F. Reid \& R. Luck \& N. Cross (Eds.), About : Designing. Analysing design meetings (pp. 269284). London : Taylor \& Francis. Aussi disponible à http://hal.archives-ouvertes.fr/inria-00410315/fr/.

Visser, W. (2009b). La conception : de la résolution de problèmes à la construction de représentations. Le Travail Humain, 72(1), 61-78. Disponible à http ://www.cairn.info/resume.php?ID_ ARTICLE=TH_721_0061. Aussi disponible à http://hal.inria.fr/inria-00410401/.

Visser, W. (2010a, February 8th-10th). Contributing to the elaboration of a design artefact according to one's interactional position: Visual and audible evidence. Paper presented at SPSD 2010, the Studying 
Professional Software Design workshop, an NSF-Sponsored International Workshop, University of California, Irvine, CA.

Visser, W. (2010b). Function and form of gestures in a collaborative design meeting. In S. Kopp \& I. Wachsmuth (Eds.), Gesture in embodied communication and human-computer interaction. 8th International Gesture Workshop, GW 2009. Bielefeld, Germany, February 25-27, 2009. Revised selected papers (Vol. LNCS 5934/2010, pp. 61-72). Heidelberg: Springer. Aussi disponible à http://hal.inria.fr/ inria-00526051.

Visser, W., \& Détienne, F. (2005). Articulation entre composantes verbale et graphico-gestuelle de l'interaction dans des réunions de conception architecturale, Actes de SCAN'05, Séminaire de Conception Architecturale Numérique : "Le rôle de l'esquisse architecturale dans le monde numérique". Charentonle-Pont, France. Disponible à http://hal.inria.fr/inria00117076/fr/.

Zemel, A., Koschmann, T., LeBaron, C., \& Feltovich, P. (2005). Usability as an interactional resource: Deictic management of scene formulation. Paper presented at CSCL '05, the 2005 conference on Computer Support for Collaborative Learning: learning 2005: the next 10 years!.

\section{RÉSUMÉ}

L'importance d'autres modes d'expression et de représentation dans l'interaction que le « verbal » a été reconnue dans le domaine du «cognitive design research $»$. À quelques exceptions près, toutefois, la seule modalité «non verbale » étudiée a été le graphique; le geste a reçu peu d'attention. Notre objectif à long terme est de déterminer la contribution de chacun des différents systèmes sémiotiques que des personnes collaborant sur une tâche adoptent dans leur interaction (modalités verbale, graphique, gestuelle et autres). Ici, nous étudions le geste dans la conception collaborative. Nous avons analysé une réunion de conception architecturale.

Notre recherche sur la conception nous avait conduit à regarder la conception comme la construction de représentations. Dans nos analyses précédentes de conception collaborative, nous y avions distingué des activités de représentation et d'organisation. Ces études étaient, toutefois, basées principalement sur du verbal.

Dans l'analyse présentée ici, nous avons observé que le geste aussi a ces deux utilisations. Il contribue à la construction de la représentation de l'artefact et à l'organisation des activités de conception et de l'interaction entre participants.

Pour un certain nombre d'auteurs dans la recherche sur le geste, parole et geste constituent un système intégré. Notre analyse confirme que les gestes sont produits principalement dans une configuration co-verbale.

Dans la conclusion, nous discutons les implications possibles de ces données pour différentes situations de travail collaboratif.

\section{MOTS-CLEFS}

Geste, interaction multimodale, conception collaborative, représentation; cognitive design research, architecture

\section{RÉFÉRENCEMENT}

Visser, W. (2011). Les gestes dans des réunions de conception architecturale. Activités, 8(2), pp. 77-99, http:// www.activites.org/v8n2/v8n2.pdf

Article soumis le 24 novembre 2010, accepté pour publication le 13 juin 2011 